OPEN ACCESS

Edited by:

Hossain Shekhar,

University of Dhaka, Bangladesh

Reviewed by:

Pedro José Carlos Rondot Radío, University of Buenos Aires, Argentina Yichao Zheng,

Tsinghua University, China

${ }^{*}$ Correspondence:

Shi Jin

catherine-jinshi@163.com

${ }^{\dagger}$ These authors have contributed equally to this work and share first authorship

Specialty section: This article was submitted to

Molecular Diagnostics and

Therapeutics,

a section of the journal

Frontiers in Molecular Biosciences

Received: 26 March 2021

Accepted: 12 October 2021

Published: 27 October 2021

Citation:

Jiao Z, Cao S, Li J, Hu N, Gong Y,

Wang $L$ and Jin $S$ (2021) Clinical

Associations of Preoperative and Postoperative Serum CEA and Lung

Cancer Outcome.

Front. Mol. Biosci. 8:686313. doi: 10.3389/fmolb.2021.686313

\section{Clinical Associations of Preoperative and Postoperative Serum CEA and Lung Cancer Outcome}

\author{
Zonglin Jiao ${ }^{1 \dagger}$, Shoubo $\mathrm{Cao}^{2 \dagger}$, Jianhua $\mathrm{Li}^{3}$, Nan $\mathrm{Hu}^{4}$, Yinghui Gong ${ }^{1}$, Linduo Wang ${ }^{1}$ and \\ Shi $\mathrm{Jin}^{5 *}$ \\ ${ }^{1}$ Department of Medical Oncology, Harbin Medical University Cancer Hospital, Harbin, China, ${ }^{2}$ Department of Medical and \\ Radiation Oncology, Linyi People's Hospital, Linyi, China, ${ }^{3}$ Department of Neurosurgery, National Cancer Center/National Clinical \\ Research Center for Cancer/Cancer Hospital and Shenzhen Hospital, Chinese Academy of Medical Sciences and Peking Union \\ Medical College, Shenzhen, China, ${ }^{4}$ Department of Oncology, Heilongjiang Agricultural Reclamation Bureau General Hospital, \\ Harbin, China, ${ }^{5}$ Department of Medical Oncology, National Cancer Center/National Clinical Research Center for Cancer/Cancer \\ Hospital and Shenzhen Hospital, Chinese Academy of Medical Sciences and Peking Union Medical College, Shenzhen, China
}

Background: Serum carcinoembryonic antigen (CEA), a classic tumour marker, is widely used in lung cancer in clinical practice. Nevertheless, few studies have elucidated the influence of dynamic changes in CEA in the perioperative phases, as a prognostic indicator, on lung cancer prognosis.

Methods: This retrospective cohort analysis included consecutive patients with stage I-III lung cancer who underwent curative resection between December 2010 and December 2014. The patients were grouped into three cohorts: group A included patients with normal preoperative CEA, group B included patients with elevated preoperative CEA but normal postoperative CEA, and group $\mathrm{C}$ included patients with elevated preoperative and postoperative CEA. Five-year overall survival (OS) was estimated by Kaplan-Meier analysis (log-rank test). Multivariate analyses were performed with Cox proportional hazard regression.

Results: A total of 1662 patients with stage I-III lung cancer were enrolled in our study. Patients with normal preoperative CEA had 15.9 and $20.1 \%$ better 3 - and 5-year OS rates than the cohort with elevated preoperative CEA $(p<0.001)$. Furthermore, group $C$ had 36.0 and $26.6 \%$ lower 5 -year OS rates $(n=74,32.4 \%)$ than group $\mathrm{A}(n=1188,68.4 \%)$ and group B $(n=139,59.0 \%)(p<0.001)$. Group B had poorer OS than group A $(p=0.016)$. For patients with different pathological TNM stages, subgroup analyses showed that group $\mathrm{C}$ had the shortest OS in stages I and II $(p<0.05)$, and patients with a postpreoperative CEA increment had poorer OS than those without an increment $(p=0.029)$. Multivariate analyses suggested that group $\mathrm{C}(\mathrm{HR}=2.0,95 \% \mathrm{Cl}, 1.5-2.7, p<0.001)$ rather than the group with normalized postoperative $\mathrm{CEA}(\mathrm{HR}=1.2,95 \% \mathrm{Cl}, 0.9-1.5, p=0.270)$ was an independent prognostic factor. In subgroup analysis of adenocarcinoma (ADC), survival analyses suggested that group $\mathrm{C}$ predicted a worse prognosis. Multivariate analysis of $A D C$ indicated that group $\mathrm{C}$ was an independent adverse prognostic factor $(\mathrm{HR}=1.9,95 \% \mathrm{Cl}, 1.4-2.7, p<0.001)$. 
Conclusions: Combined elevated preoperative and postoperative CEA is an independent adverse prognostic factor for stage I-III lung adenocarcinoma. Additionally, routine perioperative detection of serum CEA can yield valuable prognostic information for patients after lung cancer surgery.

Keywords: lung cancer, carcinoembryonic antigen, prognosis, stage I, stage II, stage III

\section{INTRODUCTION}

Cancer Statistics, 2021 indicated that in both sexes, lung cancer is a commonly diagnosed cancer ( $11.4 \%$ of total cancer cases) and the leading cause of cancer mortality $(18.0 \%$ of total cancer deaths) (Sung et al., 2021). There is no doubt that lung cancer will place a tremendous burden on society in the coming decades.

Serum tumour markers are widely used in the diagnosis and prognostic monitoring of lung cancer, and carcinoembryonic antigen (CEA) is one of the most sensitive markers (Moertel et al., 1993; Plebani et al., 1995; Molina et al., 2009). CEA is a glycoprotein associated with cell adhesion and is usually produced during foetal development but ceases to be secreted before birth. Specifically, CEA is a glycosylphosphatidylinositol (GPI) cell surface-anchored glycoprotein that serves as a ligand for L-selectin and E-selectin but is usually not present in healthy adult blood (Thomas et al., 2008; Konstantopoulos and Thomas, 2009). CEA has been widely acknowledged and recommended as a reliable tumour marker in colorectal cancer. However, it also plays vital roles in lung cancer diagnosis, progression, recurrence, metastasis, and various treatment effects (Wang et al., 2014; Chen et al., 2018; Konishi et al., 2018). Some studies have reported that a high preoperative serum CEA level is an independent prognostic factor and that a high postoperative level of serum CEA always indicates a poor prognosis in lung cancer (Sawabata et al., 2002; Sawabata et al., 2004a; Okada et al., 2004). For nonsmall lung cancer (NSCLC), in patients with a high preoperative serum CEA level, their postoperative serum CEA level has better prognostic value than their post/preoperative serum CEA ratio (Tomita et al., 2015). However, some studies have reported controversial findings on whether serum CEA can serve as a prognostic and predictive marker in lung cancer. The authors concluded that CEA is of little use as a diagnostic marker for small cell lung cancer (SCLC) and NSCLC (Ford et al., 1981; Schneider et al., 2000; Hatzakis et al., 2002).

The value of CEA in lung cancer prognosis related to dynamic changes in preoperative and postoperative serum CEA levels has not been demonstrated systematically. In our analysis, we

2477 Patients with stage I-III lung cancer during 2010-2014

815 Excluded

128 Patients with history of cancer $<5 \mathrm{y}$

432 Patients without preoperative CEA available

75 Patients with preoperative chemotherapy or RT

180 Patients without complete follow-up information

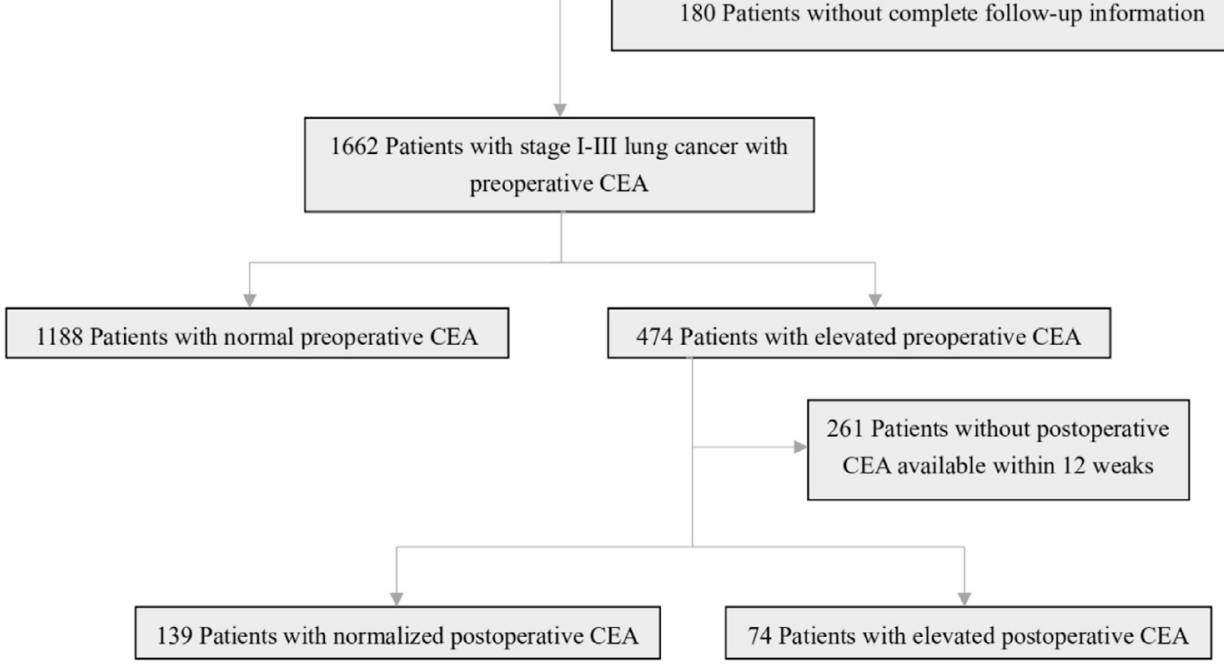

FIGURE 1 | Study design. 
TABLE 1 | Patient and tumor characteristics.

\begin{tabular}{|c|c|c|c|c|}
\hline Characteristic & All $(N=1401)$ & $\begin{array}{l}\text { Normal preoperative CEA } \\
\qquad(N=1188)\end{array}$ & $\begin{array}{l}\text { Normalized postoperative CEA } \\
\qquad(N=139)\end{array}$ & $\begin{array}{l}\text { Elevated postoperative CEA } \\
\qquad(N=74)\end{array}$ \\
\hline \multicolumn{5}{|c|}{ Gende, no. (\%) of patients } \\
\hline Male & $859(61.3)$ & $748(63.0)$ & $74(53.2)$ & $37(50.0)$ \\
\hline Female & $542(38.7)$ & $440(37.0)$ & $65(46.8)$ & $37(50.0)$ \\
\hline \multicolumn{5}{|l|}{ Age, years } \\
\hline Median (IQR) & $58(52.0-64.0)$ & $58(52.3-64.0)$ & $57(51.0-64.0)$ & $58(51.8-63.0)$ \\
\hline Mean (SD) & $57.9(8.6)$ & $58.0(8.5)$ & $55.6(9.4)$ & $57.4(7.8)$ \\
\hline \multicolumn{5}{|l|}{ Smoking } \\
\hline Yes & $793(56.6)$ & $694(58.4)$ & 69 (49.6) & $30(40.5)$ \\
\hline No & 608 (43.4) & $494(41.6)$ & $70(50.4)$ & $44(59.5)$ \\
\hline \multicolumn{5}{|l|}{ Family History } \\
\hline Yes & $230(16.4)$ & 205 (17.3) & $13(9.4)$ & $12(16.2)$ \\
\hline No & 1171 (83.6) & $983(82.7)$ & $126(90.6)$ & $62(83.8)$ \\
\hline \multicolumn{5}{|c|}{ Pathology, no. (\%) of patients } \\
\hline ADC & $864(61.7)$ & $696(58.6)$ & $102(73.4)$ & $66(89.2)$ \\
\hline SCC & $480(34.3)$ & $454(38.2)$ & $23(16.5)$ & $3(4.1)$ \\
\hline Others & $57(4.1)$ & $38(3.2)$ & $14(10.1)$ & $5(6.8)$ \\
\hline \multicolumn{5}{|c|}{ T Stage, no. (\%) of patients } \\
\hline $\mathrm{T} 1$ & 648 (46.3) & $595(50.1)$ & $38(27.3)$ & $15(20.3)$ \\
\hline T2 & $561(40.0)$ & 438 (36.9) & $79(56.8)$ & $44(59.5)$ \\
\hline T3 & $96(6.9)$ & $80(6.7)$ & $13(9.4)$ & $3(4.1)$ \\
\hline T4 & $96(6.9)$ & $75(6.3)$ & $9(6.5)$ & $12(16.2)$ \\
\hline \multicolumn{5}{|c|}{ N Stage, no. (\%) of patients } \\
\hline No & $922(65.8)$ & $837(70.5)$ & $64(46)$ & $21(28.4)$ \\
\hline N1 & $182(13)$ & $149(12.5)$ & $24(17.3)$ & $9(12.2)$ \\
\hline N2 & $294(21)$ & $199(16.8)$ & $51(36.7)$ & $44(59.5)$ \\
\hline N3 & $3(0.2)$ & $3(0.3)$ & $0(0)$ & $0(0)$ \\
\hline \multicolumn{5}{|c|}{ AJCC 7th ed. stage, no. (\%) of patients } \\
\hline IA1 & $66(4.7)$ & $66(5.6)$ & $0(0)$ & $0(0)$ \\
\hline IA2 & $235(16.8)$ & $224(18.9)$ & $9(6.5)$ & $2(2.7)$ \\
\hline IA3 & $190(13.6)$ & $178(15.0)$ & $8(5.8)$ & $4(5.4)$ \\
\hline IB & 265 (18.9) & $227(19.1)$ & $27(19.4)$ & $11(14.9)$ \\
\hline$\| A$ & $55(3.9)$ & $47(4.0)$ & $7(5)$ & $1(1.4)$ \\
\hline IIB & $219(15.6)$ & $183(15.3)$ & $31(22.3)$ & $6(8.1)$ \\
\hline IIIA & $310(22.1)$ & $221(18.6)$ & $49(35.3)$ & $40(54.1)$ \\
\hline IIIB & $61(4.4)$ & $43(3.6)$ & $8(5.8)$ & $10(13.5)$ \\
\hline \multicolumn{5}{|c|}{ Preoperative CEA, ng/ml } \\
\hline Median (IQR) & $2.7(1.8-4.1)$ & $2.4(1.6-3.4)$ & $8.6(6.2-15.1)$ & $26.9(9.6-77.5)$ \\
\hline \multicolumn{4}{|c|}{ Adjuvant chemotherapy, no. (\%) of patients } & $67.9(124.0)$ \\
\hline Yes & $560(40.0)$ & $402(33.8)$ & $109(78.4)$ & $49(66.2)$ \\
\hline No & $802(57.2)$ & $755(63.6)$ & $25(18)$ & $22(29.7)$ \\
\hline Unknown & $39(2.8)$ & $31(2.6)$ & $5(3.6)$ & $3(4.1)$ \\
\hline
\end{tabular}


TABLE 2 | Patient with postoperative CEA characteristics.

\begin{tabular}{|c|c|c|c|c|}
\hline Characteristic & All $(N=608)$ & $\begin{array}{l}\text { Normal preoperative CEA } \\
\qquad(N=395)\end{array}$ & $\begin{array}{l}\text { Normalized postoperative CEA } \\
\qquad(N=139)\end{array}$ & $\begin{array}{l}\text { Elevated postoperative CEA } \\
\qquad(N=74)\end{array}$ \\
\hline \multicolumn{5}{|c|}{ Postoperative CEA, ng/ml } \\
\hline Median (IQR) & $2.1(1.3-3.5)$ & $1.67(1.2-2.4)$ & $2.6(1.7-3.5)$ & $11.1(6.4-31.6)$ \\
\hline Mean (SD) & $10.6(63.5)$ & $3.8(15.7)$ & $2.6(1.1)$ & $62.0(170.6)$ \\
\hline \multicolumn{5}{|c|}{$\begin{array}{l}\text { Days from surgery to CEA } \\
\text { testing }\end{array}$} \\
\hline Median (IQR) & $\begin{array}{c}38 \\
(32.0-54.8)\end{array}$ & $38(32.0-54.0)$ & 37 (32.0-55.0) & $39.5(33.0-54.3)$ \\
\hline Mean (SD) & $43.7(16.1)$ & $43.5(16.4)$ & $43.7(15.6)$ & $44.6(16.0)$ \\
\hline
\end{tabular}
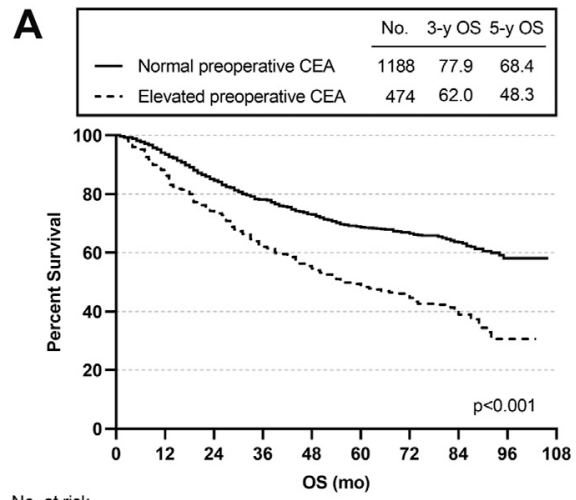

$\begin{array}{llllllllll}\text { No. at risk } & & & & & & & & & \\ \text { Normal } & 1188 & 1115 & 1009 & 926 & 867 & 817 & 494 & 257 & 48\end{array}$ $\begin{array}{lrrrrrrrrr}\text { Normal } & 1188 & 1115 & & & & & & & \\ \text { Elevated } & 474 & 418 & 352 & 296 & 263 & 234 & 147 & 67 & 7\end{array}$

C
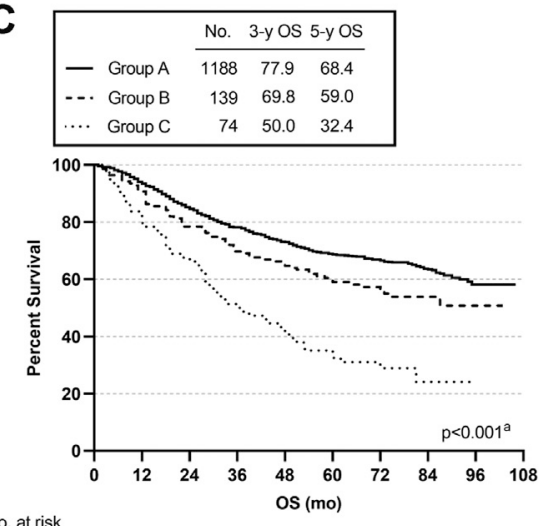

No. at risk
Normal

$\begin{array}{lrrrrrrrr}1188 & 1115 & 1009 & 926 & 867 & 817 & 494 & 257 & 48\end{array}$

$\begin{array}{lrrrrrrrrr}\text { Normalized } & 139 & 127 & 109 & 97 & 91 & 83 & 51 & 27 & 3 \\ \text { Elevated } & 74 & 62 & 50 & 38 & 32 & 26 & 15 & 4 & 1\end{array}$

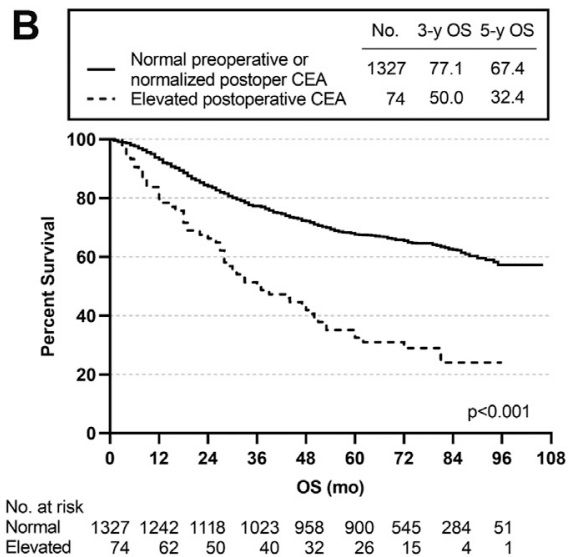

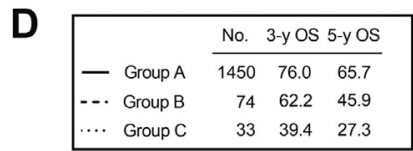

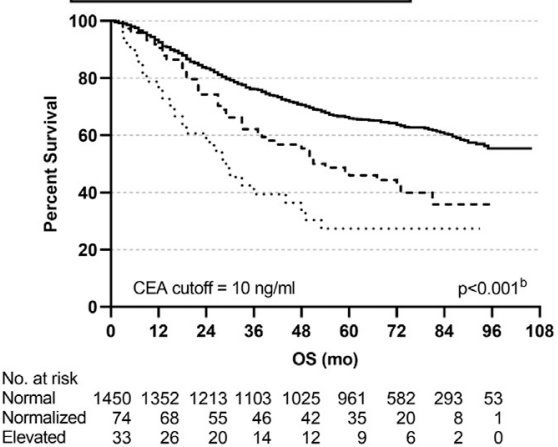

FIGURE 2 I Overall survival (OS) curves according to preoperative and postoperative CEA level. (A) K-M curve for OS in patients with normal vs. elevated preoperative CEA. (B) K-M curve for OS in patients with normal preoperative or normalized postoperative CEA vs. elevated postoperative CEA. (C) K-M curve for OS in patients with different subgroups. (D) K-M curve for OS in patients with different subgroups using a CEA cut-off of 10 ng/ml. ${ }^{a} \mathrm{Group} \mathrm{A} \mathrm{vs.} \mathrm{Group} \mathrm{B,} p=0.016$; Group A vs. Group C, $p<0.001$; Group B vs. Group C, $p<0.001$. ' Group A vs. Group B, $p<0.001$; Group A vs. Group C, $p<0.001$; Group B vs. Group C, $p=0.048$.

attempted to observe whether perioperative changes in CEA could provide more prognostic information. More specifically, we sought to explore whether patients with an elevated preoperative CEA level that normalizes after curative resection have a similar risk of death as those with a CEA level that is elevated throughout the perioperative period.

\section{METHODS}

\section{Study Design and Data Collection}

This study was a retrospective clinical study and was approved by the Ethics Committee of Harbin Medical University Cancer Hospital. All the consecutive patients were from the Harbin 

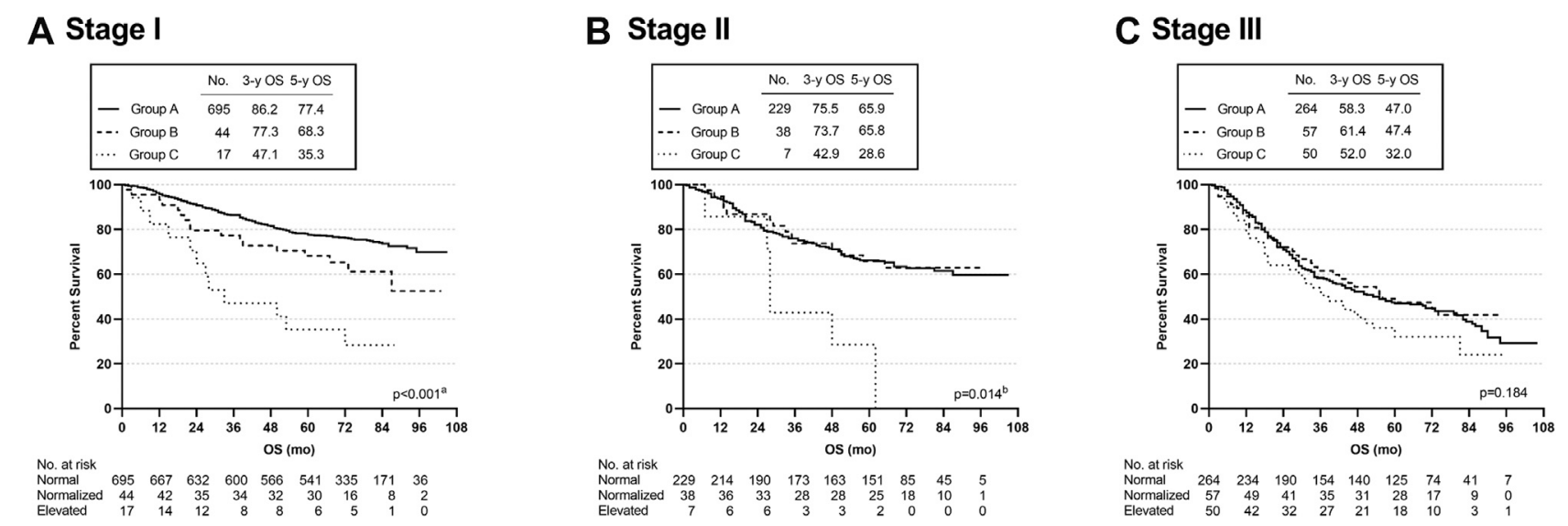

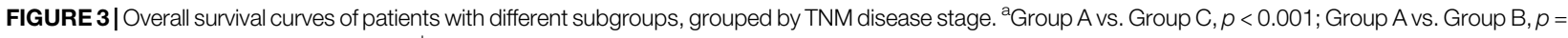
0.039; Group B vs. Group C, $p=0.018$. ' Group A vs. Group C, $p=0.004$; Group B vs. Group C, $p=0.014$.

Medical University Cancer Hospital and pathologically diagnosed with stage I to III lung cancer between December 2010 and December 2014. The exclusion criteria were as follows: 1) treatment for malignancy within 5 years; 2) no available preoperative CEA value; 3) preoperative chemotherapy or radiotherapy; and 4) a lack of complete follow-up information.

Data on the patients' clinical characteristics, including demographics, pathological reports, and perioperative clinical outcomes, were acquired from the departmental database and electronic patient records. Pathological stage was defined based on the seventh edition of the AJCC lung cancer criteria. We defined preoperative CEA as the CEA value recorded closest to the surgery time, and postoperative CEA as the CEA value recorded within 12 weeks after surgery but before postoperative adjuvant therapy. The normal reference CEA value for the assay was $0.0-5.0 \mathrm{ng} / \mathrm{ml}$ (Roche Cobas e $602 \mathrm{ECL}$ analyser). Patients were grouped as follows: 1) (Group A) normal preoperative CEA group, patients with a normal preoperative CEA level ( $\leq 5 \mathrm{ng} / \mathrm{ml}$ ); 2) (Group B) normalized postoperative CEA group, patients with an elevated preoperative CEA level (>5 ng/ml) but a normal postoperative CEA level; and 3) (Group C) elevated postoperative CEA group, patients with elevated preoperative and postoperative CEA levels. We also used a CEA cut-off of $10.0 \mathrm{ng} / \mathrm{ml}$ for repeat analyses (Konishi et al., 2018). According to authoritative guidelines, all patients were followed up by history, physical examination, chest CT and PET/ CT or MRI. Survival statuses were effectively updated by telephone, email, or medical history. Overall survival (OS) was defined as the time from surgery to death or last follow-up. Patients alive at the last follow-up date were censored.

\section{Statistical Analysis}

All statistical data in this study were analysed with IBM SPSS 21.0 statistical software and GraphPad Prism 8.0. Three- and 5-year overall survival (OS) was estimated by the Kaplan-Meier method with the log-rank test for univariate analysis. The various indicators of prognosis with $p$-values of less than 0.05 in the univariate analysis were examined in multivariate analyses.
Hazard ratios (HRs), 95\% confidence intervals (CIs), and the effects of prognostic factors were estimated by Cox regression in the multivariate Cox regression. All $p$-values were two-sided, and $p$-values $<0.05$ were considered significant.

\section{RESULTS}

A total of 2477 consecutive patients with stage I-III lung cancer who underwent curative resection were included in our study. According to the exclusion criteria, patients who had prior cancer treatment within 5 years $(n=128)$, were missing a preoperative CEA value $(n=432)$, had received preoperative chemotherapy or radiotherapy $(n=75)$, or had incomplete follow-up information $(n=180)$ were excluded. Among the remaining 1662 patients, $1188(71.5 \%)$ had a normal preoperative CEA level, and 474 (28.5\%) had an elevated CEA level. A total of 261 of the 474 patients with an elevated preoperative CEA level had no available postoperative CEA data within 12 weeks; 139 of the remaining patients had a normalized postoperative CEA value, and 74 had an elevated postoperative CEA value (Figure 1).

Descriptive statistics of patient and tumour characteristics for 1401 patients are shown in Table 1. There were 859 males (61.3\%) and 542 females $(38.7 \%)$ in our study. The median age (IQR) of all these patients was $58(52-64)$ years (mean age, $57.9 \pm 8.6$ years). More than half of the patients $(56.6 \%$, $n=796)$ had a smoking history. Most patients $(83.6 \%, n=1171)$ did not have a family history of cancer. The main types of pathology included adenocarcinoma (ADC; 61.7\%, $n=864$ ), squamous cell carcinoma (SCC; $34.3 \%, n=480)$ and others $(4.1 \%, n=57)$. For $\mathrm{T}$ stage, 648 patients $(46.3 \%)$ had $\mathrm{T} 1$ disease, and $561(40.0 \%)$ had T2 disease, while there were only 96 in stage T3 or T4. A total of 479 patients had lymph node metastasis, including 182 patients (13\%) with N1 disease, 294 (21\%) with N2 disease and $3(0.2 \%)$ with N3 disease. The median (IQR) preoperative CEA level was $2.7(1.8-4.1) \mathrm{ng} / \mathrm{ml}$. In 608 patients with postoperative CEA data, the median postoperative CEA level was $2.1(1.3-3.5) \mathrm{ng} / \mathrm{ml}$, and the median (IQR) days 


\section{A ADC}
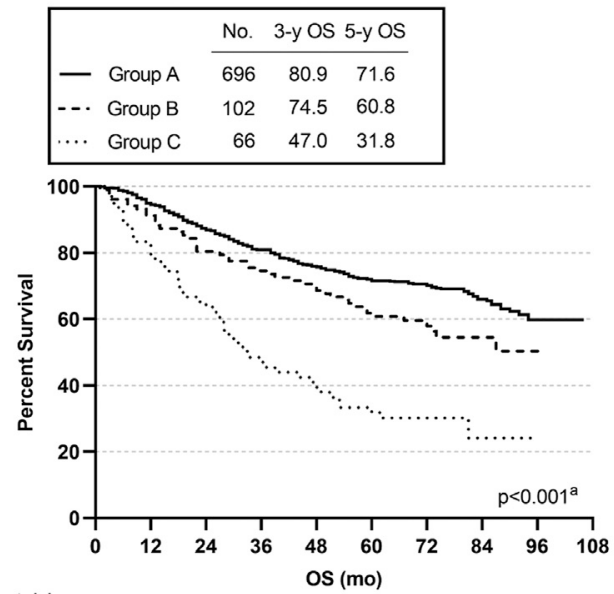

No. at risk

$\begin{array}{llllllllll}\text { Normal } & 696 & 661 & 608 & 563 & 528 & 501 & 300 & 144 & 32\end{array}$

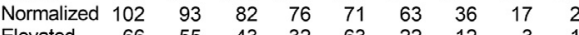

\section{Others}

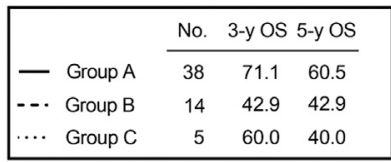

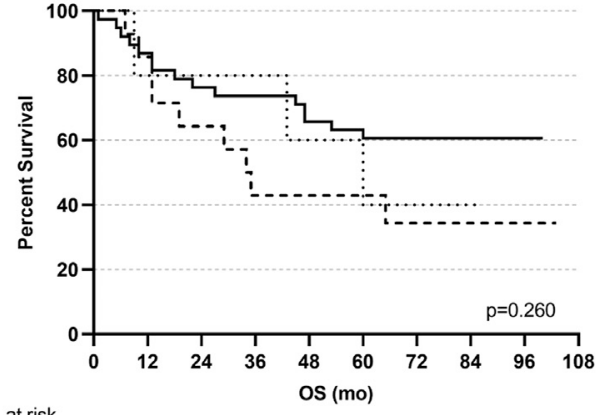

No. at risk

$\begin{array}{llllllllll}\text { Normal } & 38 & 33 & 29 & 28 & 25 & 24 & 18 & 11 & 2\end{array}$

$\begin{array}{lrrrrrrrrr}\text { Normalized } & 14 & 12 & 9 & 6 & 6 & 6 & 4 & 3 & 1 \\ \text { Elevated } & 5 & 4 & 4 & 4 & 3 & 3 & 2 & 1 & 0\end{array}$
B SCC
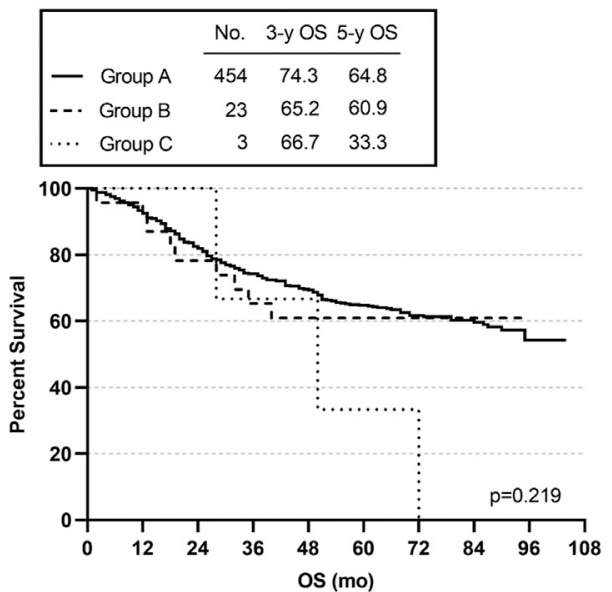

No. at risk

$\begin{array}{llllllllll}\text { Normal } & 454 & 421 & 372 & 336 & 314 & 292 & 176 & 102 & 14\end{array}$ $\begin{array}{lrrrrrrrrr}\text { Normalized } & 23 & 22 & 18 & 15 & 14 & 14 & 11 & 7 & 0 \\ \text { Elevated } & 3 & 3 & 3 & 2 & 2 & 1 & 1 & 0 & 0\end{array}$

D ADC

\begin{tabular}{|c|c|c|c|c|}
\hline \multirow[b]{2}{*}{ - } & \multirow[b]{2}{*}{ Group A } & \multicolumn{3}{|c|}{ No. 3-y OS 5-y OS } \\
\hline & & 878 & 78.5 & 68.0 \\
\hline-- & Group B & 64 & 59.4 & 45.3 \\
\hline$\ldots$ & Group C & 30 & 36.7 & 23.3 \\
\hline
\end{tabular}

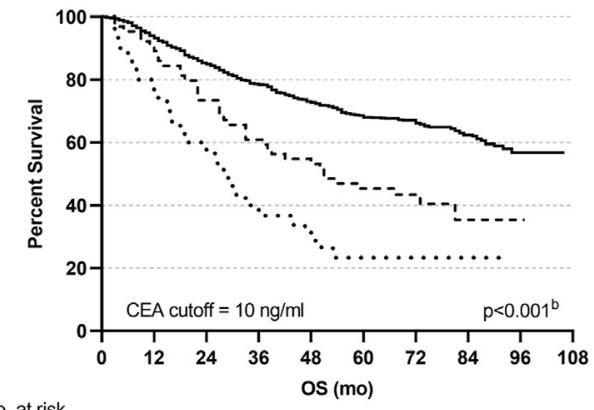

$\begin{array}{llllllllll}\text { No. at risk } & & & & & & & & & \\ \text { Normal } & 878 & 825 & 749 & 690 & 641 & 602 & 359 & 165 & 36\end{array}$

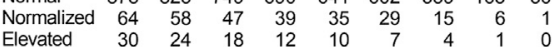

FIGURE 4 | Overall survival curves of patients with different subgroups, grouped by pathological types. (A-C) K-M curves for OS in ADC, SCC, and Others. (D) K-M curve for OS in ADC using a CEA cut-off of 10 ng/ml. ${ }^{a}$ Group A vs. Group C, $p<0.001$; Group A vs. Group B, $p=0.014$; Group B vs. Group C, $p<0.001$. ${ }^{b}$ Group A vs. Group B, $p<0.001$; Group A vs. Group C, $p<0.001$; Group B vs. Group C, $p=0.029$.

from surgery to CEA testing was 38 (32.0-54.8) days (Table 2). The median (IQR) follow-up time was 76 (67-87) months. According to our follow-up data, a total of 537 patients died. The 5-year OS rate for all these patients was $65.6 \%$.

Kaplan-Meier survival curves were used to assess the effect of different CEA groups. The 3- and 5-year OS rates for the 1188 patients with a normal preoperative CEA level were 77.9 and $68.4 \%$, which were much higher than the corresponding OS rates of 62 and $48.3 \%$ for the 474 patients with an elevated preoperative CEA level $(p<0.001)$ (Figure 2A). The 3- and 5-year OS rates for the 74 patients whose CEA value remained elevated after surgery at 12 weeks were 50 and $32.4 \%$ compared with 77.1 and $67.4 \%$ for the 1327 patients with either a normalized postoperative CEA level $(n=139)$ or a normal preoperative CEA level $(n=1188)$ $(p<0.001)$ (Figure 2B). The 3- and 5-year OS rates were 69.8 and $59 \%$ for the 139 patients in group B, which were significantly different from those in group A and group $C$ (group A vs. group $\mathrm{B}, p=0.016$; group $\mathrm{A}$ vs. group $\mathrm{C}, p<0.001$; group $\mathrm{B}$ vs. group $\mathrm{C}$, $p<0.001$ ) (overall log-rank $p<0.001$ ) (Figure 2C). Moreover, using a CEA cut-off of $10.0 \mathrm{ng} / \mathrm{ml}$ for repeat analyses produced similar results. Group A had the best prognosis, while group $\mathrm{C}$ had the worst (group A vs. group B, $p<0.001$; group A vs. group $\mathrm{C}, p<0.001$; group B vs. group $\mathrm{C}, p=0.048$ ) (overall $\log$-rank $p<$ 0.001) (Figure 2D).

Subgroup analyses were used to assess the effect of CEA levels on specific stages. In stage I, the OS rate was significantly different among the three cohorts $(p<0.001)$; in particular, the 3 -year and 5 -year OS rates of group $C$ were observably lower than those of 


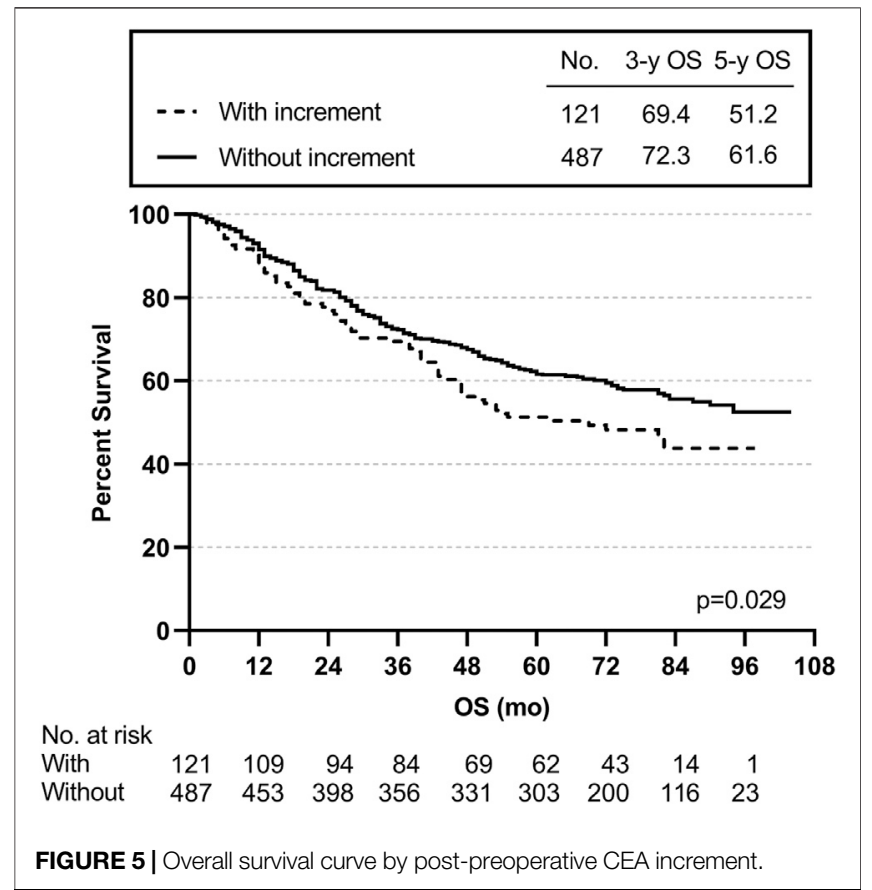

the other two groups (group A vs. group $\mathrm{B}, p=0.039$; group A vs. group $C, p<0.001$; group $B$ vs. group $C, p=0.018$ ) (Figure $3 \mathrm{~A}$ ). In patients with stage II disease, OS was significantly lower in group $\mathrm{C}$ than in group $\mathrm{A}$ or group $\mathrm{B}$ (group $\mathrm{A}$ vs. group $\mathrm{C}, p=$ 0.004; group B vs. group $C, p=0.014$ ) (overall log-rank $p<0.001$ ) (Figure 3B). In stage III, the three cohorts did not differ significantly ( $p=0.184)$ (Figure 3C).

Subgroup analysis was conducted according to the pathological type of lung cancer. The 3- and 5-year OS rates of the ADC patients in group B were $74.5 \%$ and 60.8 , respectively, and there were statistically significant differences among the three groups (group A vs. group $\mathrm{B}, p=0.014$; group $\mathrm{A}$ vs. group $\mathrm{C}, p<$ 0.001 ; group $B$ vs. group $C, p<0.001$ ) (overall log-rank $p<0.001$ )

(Figure 4A). However, there were no significant differences in OS between SCC and other subtypes of lung cancer (Figures 4B,C). Furthermore, using a CEA cut-off of $10.0 \mathrm{ng} / \mathrm{ml}$ for repeat analyses in ADC produced similar results (group A vs. group $\mathrm{B}, p<0.001$; group $\mathrm{A}$ vs. group $\mathrm{C}, p<0.001$; group $\mathrm{B}$ vs. group $\mathrm{C}$, $p=0.029$ ) (overall log-rank $p<0.001$ ) (Figure 4D). Similarly, there were no statistically significant differences in OS between SCC and other pathological subtypes using a CEA cut-off of $10.0 \mathrm{ng} / \mathrm{ml}$. The above analysis results suggested that the OS differences among the three groups (groups A, B, and C) defined by our inclusion data might be mainly reflected in lung adenocarcinoma.

We also created subgroups to test the effect of postpreoperative CEA on prognosis. The post-preoperative CEA increment meant that the postoperative CEA level was higher than the preoperative CEA level. The 3- and 5-year OS rates for the 121 patients with a post-preoperative CEA increment were

TABLE 3 | Univariate and multivariate analyses of overall survival.

\begin{tabular}{|c|c|c|c|c|c|c|}
\hline \multirow[t]{2}{*}{ Variable } & \multicolumn{3}{|c|}{ Univariate } & \multicolumn{3}{|c|}{ Multivariate } \\
\hline & 5-year OS (\%) & $95 \% \mathrm{Cl}$ & $p$ & Hazard ratio & $95 \% \mathrm{Cl}$ & $p$ \\
\hline Gender & & & 0.001 & & & \\
\hline Female & 71.6 & $67.9-75.3$ & & 0.7 & $0.6-0.9$ & 0.005 \\
\hline Age & & & 0.285 & & & \\
\hline Smoking & & & 0.026 & & & \\
\hline No & 69.6 & $65.9-73.3$ & & Ref & & \\
\hline Yes & 62.5 & $59.2-65.8$ & & 1.1 & $0.9-1.4$ & 0.268 \\
\hline Family History & & & 0.667 & & & \\
\hline No & 65.3 & $62.6-68.0$ & & & & \\
\hline SCC & 64.0 & $59.7-68.3$ & & & & \\
\hline Others & 54.4 & $41.5-67.3$ & & & & \\
\hline TNM stage & & & $<0.001$ & & & \\
\hline । & 75.9 & $72.8-79.0$ & & Ref & & \\
\hline$\|$ & 65.0 & $59.3-70.7$ & & 1.5 & $1.2-1.9$ & 0.002 \\
\hline III & 45.0 & $39.9-50.1$ & & 2.7 & $2.2-3.2$ & $<0.001$ \\
\hline CEA group & & & $<0.001$ & & & \\
\hline Normal preoperative CEA (A) & 68.4 & $65.9-70.9$ & & Ref & & \\
\hline Normalized Postoperative CEA (B) & 59.0 & $50.8-67.2$ & & 1.2 & $0.9-1.5$ & 0.270 \\
\hline Elevated Postoperative CEA ${ }^{\odot}$ & 32.4 & $21.8-43.0$ & & 2.0 & $1.5-2.7$ & $<0.001$ \\
\hline
\end{tabular}


TABLE 4 | Univariate and multivariate analyses of overall survival in ADC.

\begin{tabular}{|c|c|c|c|c|c|c|}
\hline \multirow[t]{2}{*}{ Variable } & \multicolumn{3}{|c|}{ Univariate } & \multicolumn{3}{|c|}{ Multivariate } \\
\hline & 5-year OS (\%) & $95 \% \mathrm{Cl}$ & $p$ & Hazard ratio & $95 \% \mathrm{Cl}$ & $p$ \\
\hline Gender & & & 0.002 & & & \\
\hline Male & 62.0 & $57.3-66.7$ & & Ref & & \\
\hline Female & 72.0 & $67.9-76.1$ & & 0.7 & $0.6-0.9$ & 0.001 \\
\hline Age & & & 0.335 & & & \\
\hline$<65$ & 68.0 & $64.5-71.5$ & & & & \\
\hline$\geq 65$ & 64.4 & $57.3-71.5$ & & & & \\
\hline Smoking & & & 0.183 & & & \\
\hline No & 69.4 & $65.5-73.3$ & & & & \\
\hline Yes & 64.1 & $59.2-69.0$ & & & & \\
\hline Family History & & & 0.341 & & & \\
\hline No & 66.8 & $63.5-70.1$ & & & & \\
\hline Yes & 70.8 & $62.2-79.4$ & & & & \\
\hline TNM stage & & & $<0.001$ & & & \\
\hline I & 78.1 & $74.6-81.6$ & & Ref & & \\
\hline$\|$ & 62.0 & $53.4-70.6$ & & 1.8 & $1.3-2.5$ & 0.001 \\
\hline III & 45.4 & $38.9-51.9$ & & 3.1 & $2.4-4.0$ & $<0.001$ \\
\hline CEA group & & & $<0.001$ & & & \\
\hline Normal preoperative CEA (A) & 71.6 & $68.3-74.9$ & & Ref & & \\
\hline Normalized Postoperative CEA (B) & 60.8 & $51.4-70.2$ & & 1.1 & $0.8-1.5$ & 0.676 \\
\hline Elevated Postoperative CEA (C)) & 31.8 & $20.6-43.0$ & & 1.9 & $1.4-2.7$ & $<0.001$ \\
\hline
\end{tabular}

69.4 and $51.2 \%$ lower than the rates of 72.3 and $61.6 \%$ for the 487 patients without a post-preoperative CEA increment $(p=0.029)$ (Figure 5).

The results for univariate and multivariate analyses revealing the clinical factors associated with OS are shown in Table 3. Using K-M curves in the univariate analysis, our results suggested that sex, smoking history, TNM (tumour-node-metastasis) stage, and different groups of changes in CEA levels were associated with OS. However, age, family cancer history, and pathology had no significance for OS. Furthermore, the multivariate analyses indicated that an elevated postoperative CEA level was an independent prognostic factor for OS $(\mathrm{HR}=2.0,95 \% \mathrm{CI}$, 1.5-2.7, $p<0.001$ ) rather than a normalized postoperative CEA level $(\mathrm{HR}=1.2,95 \%$ CI\%, 0.9-1.5, $p=0.270)$. Additionally, shorter OS was associated with male sex and a higher TNM stage.

In the subgroup analysis, we found that the differences in OS among the three groups might be mainly reflected in ADC. Therefore, univariate and multivariate analyses were conducted in patients with ADC. Our results indicated that sex, TNM stage, and different groups of changes in CEA levels were associated with OS. Moreover, the multivariate analyses suggested that an elevated postoperative CEA level was an independent prognostic factor for OS (HR $=1.9,95 \%$ CI, 1.4-2.7, $p<0.001)$ rather than a normalized postoperative CEA level $(\mathrm{HR}=1.1,95 \% \mathrm{CI} \%, 0.8-1.5, p=0.676)$. Additionally, shorter OS was closely associated with male sex and a higher TNM stage (Table 4).

\section{DISCUSSION}

In our study, the data showed that changes in the perioperative CEA level provided an informative prognostic reference for patients with stage I-III lung cancer who underwent curative resection. Patients with a normal preoperative CEA level had 15.9 and $20.1 \%$ higher 3- and 5-year OS rates than those with an elevated preoperative CEA level, and this result was consistent with the literature (Icard et al., 1994; Foa et al., 1999; Suzuki et al., 1999; Okada et al., 2003; Matsuoka et al., 2007). Following surgery, more than $65 \%$ of patients had a normalized CEA level, and the outcomes of these patients were worse than those of patients with a normal preoperative CEA level but better than those of patients with an elevated postoperative CEA level. Repeat analyses using a CEA cut-off of $10.0 \mathrm{ng} / \mathrm{ml}$ obtained similar results. Furthermore, those patients with an elevated CEA level following surgery had an absolute 27.1 and $35 \%$ lower 3- and 5-year OS rates than those with either a normalized postoperative CEA level or a normal preoperative CEA level. Subgroup analyses clearly showed a significant difference in survival among the three groups in stage I, with similar trends in stages II, and revealed poor survival in patients with a post-preoperative CEA increment. However, perioperative CEA is not able to stratify patients with stage III disease, likely due to the high tumor load of stage III patients and the early recurrence and metastasis. Moreover, in our data, fewer patients were included in group $\mathrm{B}$ and group $\mathrm{C}$, which may overestimate the survival rate of these two groups. Univariate 
analysis suggested that sex, smoking history, TNM stage, and different groups of changes in CEA levels were related to OS. In addition, multivariable analyses demonstrated that a persistently elevated CEA level following surgery was an independent prognostic factor for lung cancer, consistent with previous research (Wang et al., 2010; Kozu et al., 2013; Duan et al., 2015). We found that lung adenocarcinoma accounted for the majority $(61.7 \%)$ of all pathological subtypes we enrolled. We conducted survival analysis according to different pathological subtypes by the K-M method and found that only lung adenocarcinoma had results similar to those reported above. Furthermore, univariate and multivariate analyses found that an elevated postoperative CEA level was an independent prognostic factor in lung adenocarcinoma. Using a CEA cutoff of $10 \mathrm{ng} / \mathrm{ml}$, consistent trend results were obtained. This indicated that the positive results obtained in all patients enrolled in our study were probably due to the large proportion of lung adenocarcinoma patients.

The findings are similar to those of other studies (Sawabata et al., 2002; Okada et al., 2004); evaluating perioperative serum CEA levels in patients with lung cancer following surgery has significant prognostic value. In most patients, preoperative CEA levels returned to normal after surgery. These patients' survival status was significantly better than that of patients whose postoperative CEA level remained elevated, indicating that a normalized postoperative CEA level is an essential and favourable prognostic indicator for patients with a higher preoperative CEA level than normal. We concluded that patients with persistent CEA elevation have the worst prognosis even after apparent surgical success and require more careful follow-up. Another study noted that patients with low levels of postoperative CEA $(<2.5 \mathrm{ng} / \mathrm{ml})$ had an extremely favourable prognosis compared with those with normal or elevated postoperative CEA levels among patients with pathologic stage Ia NSCLC (Sawabata et al., 2004b). However, some studies have reached the controversial conclusion that CEA is not useful as a diagnostic marker in lung cancer (Hanagiri et al., 2011; Takahashi et al., 2011). Therefore, these data must be interpreted with care. CEA may carry prognostic information, but it might not be an adequate prognostic indicator for guiding clinical decisions. The conflicting results may indicate that serum CEA alone is not sufficiently sensitive for monitoring patient outcomes; however, the combination of CT findings and pre/ postoperative serum CEA levels provides reliable prognostic information for DFS and OS in lung cancer patients (Takamochi et al., 2004; Higashi et al., 2009; Yamazaki et al., 2015). In addition, the combined detection of serum tumour markers before lung cancer surgery has significant prognostic value. For example, combined detection of CEA and CYFRA21-1, tumour marker indexes, may be a promising approach for assessing patient prognosis (Muley et al., 2008; Tomita et al., 2010).

As a tumour marker with a prognostic role in lung cancer, CEA is convenient to detect during preoperative or postoperative periods and has been widely used in clinical practice. However, guidelines published by the American Thoracic Society and European Respiratory Society stated that CEA routine testing was not recommended for staging or disease prognosis (Jett et al., 1997). Furthermore, the NCCN guidelines for the management of NSCLC issued in 2021 did not recommend CEA as a pretreatment evaluation indicator (NCNN, 2020). Nevertheless, Ozeki et al. emphasized the importance of follow-up by CEA monitoring for patients after lung cancer surgery (Ozeki et al., 2014). In clinical practice, should we refer to perioperative serum CEA levels or dynamic changes in CEA levels to provide treatment or follow-up recommendations for patients with lung cancer? We believe that serum tumour markers play an important role in lung cancer, but these markers have not received enough attention in clinical practice. Therefore, our study may provide meaningful insights for clinicians. Of course, there were some limitations to our study. First, this was a retrospective study subject to the inherent limitations and biases of observational retrospective research. For example, the number of patients with a normalized postoperative CEA level or an elevated postoperative CEA level was smaller than that of patients with a normal preoperative CEA level. Second, the limitations of this observational study were typical for cohorts of patients treated at a single academic institution, including potential selection biases, generalizability, and attrition (only half of the patients with an elevated CEA level at baseline had postoperative CEA information). In comparison, prospective studies will provide more convincing evidence of the significance of CEA in lung cancer and these data need to be further verified by multiple centres. In addtion, in our study, some factors were missing or not recorded, such as comorbidities and economic status, so the suggestive effect of perioperative detection of serum CEA on the risk of death may be overestimated, and its real effect needs to be further studied. Again, we studied only one tumour marker, and the combination of multiple indicators (other tumor biomarkers or imaging methods) might provide more clinical significance. Finally, the patients in our research were all Chinese.

In conclusion, our results demonstrate the effect of dynamic changes in serum preoperative and postoperative CEA levels in a large cohort of patients undergoing resection for lung cancer. They indicate that the postoperative CEA level may inform the frequency of surveillance. Therefore, this study will provide valuable information for lung cancer patients as a clinical reference for follow-up treatment.

\section{CONCLUSION}

Patients with a normalized postoperative CEA level or an elevated postoperative CEA level had worse OS than those with a normal preoperative level. Unlike a normalized postoperative CEA level, an elevated postoperative CEA level is an independent adverse prognostic factor in lung 
cancer patients undergoing surgery, especially in lung adenocarcinoma. It is emphasized that CEA monitoring during the perioperative period will provide more valuable prognostic information for patients with lung adenocarcinoma.

\section{DATA AVAILABILITY STATEMENT}

The original contributions presented in the study are included in the article/Supplementary Materials, further inquiries can be directed to the corresponding author.

\section{ETHICS STATEMENT}

The studies involving human participants were reviewed and approved by Ethics Committee of Harbin Medical University Cancer Hospital. The patients/participants provided their written informed consent to participate in this study. Written informed consent was obtained from the individual(s) for the publication of any potentially identifiable images or data included in this article.

\section{REFERENCES}

Chen, Z.-q., Huang, L.-s., and Zhu, B. (2018). Assessment of Seven Clinical Tumor Markers in Diagnosis of Non-small-cell Lung Cancer. Dis. Markers 2018, 1-7. doi:10.1155/2018/9845123

Cui, Y., Duan, X., Li, H., Shi, G., Wu, B., Liu, M., et al. (2015). High Preoperative and Postoperative Levels of Carcinoembryonic Antigen and CYFRA 21-1 Indicate Poor Prognosis in Patients with Pathological Stage I Nonsmall Cell Lung Cancer. Indian J. Cancer 52 (Suppl. 3), 158-E163. doi:10.4103/0019509X.186564

Foa, P., Fornier, M., Miceli, R., Seregni, E., Santambrogio, L., Nosotti, M., et al. (1999). Tumour Markers CEA, NSE, SCC, TPA and CYFRA 21.1 in Resectable Non-small Cell Lung Cancer. Anticancer Res. 19 (4C), 3613-3618.

Ford, C. H., Stokes, H. J., and Newman, C. E. (1981). Carcinoembryonic Antigen and Prognosis after Radical Surgery for Lung Cancer: Immunocytochemical Localization and Serum Levels. Br. J. Cancer 44, 145-153. doi:10.1038/ bjc. 1981.164

Hanagiri, T., Sugaya, M., Takenaka, M., Oka, S., Baba, T., Shigematsu, Y., et al. (2011). Preoperative CYFRA 21-1 and CEA as Prognostic Factors in Patients with Stage I Non-small Cell Lung Cancer. Lung Cancer 74 (1), 112-117. doi:10.1016/j.lungcan.2011.02.001

Hatzakis, K. D., Froudarakis, M. E., Bouros, D., Tzanakis, N., Karkavitsas, N., and Siafakas, N. M. (2002). Prognostic Value of Serum Tumor Markers in Patients with Lung Cancer. Respiration 69, 25-29. doi:10.1159/000049366

Higashi, K., Sakuma, T., Ito, K., Niho, S., Ueda, Y., Kobayashi, T., et al. (2009). Combined Evaluation of Preoperative FDG Uptake on PET, Ground-Glass Opacity Area on CT, and Serum CEA Level: Identification of Both Low and High Risk of Recurrence in Patients with Resected T1 Lung Adenocarcinoma. Eur. J. Nucl. Med. Mol. Imaging 36 (3), 373-381. doi:10.1007/s00259-0080961-4

Icard, P., Regnard, J.-F., Essomba, A., Panebianco, V., Magdeleinat, P., and Levasseur, P. (1994). Preoperative Carcinoembryonic Antigen Level as a Prognostic Indicator in Resected Primary Lung Cancer. Ann. Thorac. Surg. 58 (3), 811-814. doi:10.1016/0003-4975(94)90755-2

Jett, J., Feins, R., Kvale, P., Mccloud, T., Miller, Y. W., Perry, M., et al. (1997). Pretreatment Evaluation of Non-small-cell Lung Cancer. The American

\section{AUTHOR CONTRIBUTIONS}

SJ and ZJ designed the study concept and took responsibility for the integrity of the data. ZJ analyzed all data and drafted the manuscript. SC designed the study and revised the manuscript. JL revised the manuscript and supervised the study. $\mathrm{NH}, \mathrm{YG}$, and LW collected clinical and follow-up data from the Harbin Medical University Cancer Hospital.

\section{FUNDING}

The work was supported by Shenzhen Science and Technology Program (Grant No. RCJC20200714114436049) and Cancer Hospital, Chinese Academy of Medical Sciences, Shenzhen Center/ShenZhen Cancer Hospital Research Project (No. SZ2020ZD006).

\section{ACKNOWLEDGMENTS}

We thank the ethics committee and the electronic medical record department sincerely.

Thoracic Society and the European Respiratory Society. Am. J. Respir. Crit. Care Med. 156 (1), 320-332. doi:10.1164/ajrccm.156.1.ats156.1

Konishi, T., Shimada, Y., Hsu, M., Tufts, L., Jimenez-Rodriguez, R., Cercek, A., et al. (2018). Association of Preoperative and Postoperative Serum Carcinoembryonic Antigen and Colon Cancer Outcome. JAMA Oncol. 4 (3), 309. doi:10.1001/jamaoncol.2017.4420

Konstantopoulos, K., and Thomas, S. N. (2009). Cancer Cells in Transit: The Vascular Interactions of Tumor Cells. Annu. Rev. Biomed. Eng. 11, 177-202. doi:10.1146/annurev-bioeng-061008-124949

Kozu, Y., Maniwa, T., Takahashi, S., Isaka, M., Ohde, Y., and Nakajima, T. (2013). Prognostic Significance of Postoperative Serum Carcinoembryonic Antigen Levels in Patients with Completely Resected Pathological-Stage I Non-small Cell Lung Cancer. J. Cardiothorac. Surg. 8, 106. doi:10.1186/1749-8090-8-106

Matsuoka, K., Sumitomo, S., Nakashima, N., Nakajima, D., and Misaki, N. (2007). Prognostic Value of Carcinoembryonic Antigen and CYFRA21-1 in Patients with Pathological Stage I Non-small Cell Lung Cancer. Eur. J. Cardio-Thoracic Surg. 32 (3), 435-439. doi:10.1016/j.ejcts.2007.05.014

Moertel, C. G., Fleming, T. R., Macdonald, J. S., Haller, D. G., Laurie, J. A., and Tangen, C. (1993). An Evaluation of the Carcinoembryonic Antigen (CEA) Test for Monitoring Patients with Resected Colon Cancer. JAMA 270 (8), 943-947. doi:10.1001/jama.270.8.943

Molina, R., Augé, J. M., Bosch, X., Escudero, J. E. M., Viñolas, N., Marrades, R. O. N., et al. (2009). Usefulness of Serum Tumor Markers, Including ProgastrinReleasing Peptide, in Patients with Lung Cancer: Correlation with Histology. Tumor Biol. 30, 121-129. doi:10.1159/000224628

Muley, T., Fetz, T.-H., Dienemann, H., Hoffmann, H., Herth, F. J. F., Meister, M., et al. (2008). Tumor Volume and Tumor Marker Index Based on CYFRA 21-1 and CEA Are strong Prognostic Factors in Operated Early Stage NSCLC. Lung Cancer 60 (3), 408-415. doi:10.1016/j.lungcan.2007.10.026

NCCN. 2020). Clinical Practice Guideline in Oncology, Non-small-cell Lung Cancer. Version 6.2020. Pennsylvania: NCCN.

Okada, M., Sakamoto, T., Nishio, W., Uchino, K., and Tsubota, N. (2003). Characteristics and Prognosis of Patients After Resection of Nonsmall Cell Lung Carcinoma Measuring $2 \mathrm{Cm}$ or Less in Greatest Dimension. Cancer 98 (3), 535-541. doi:10.1002/cncr.11530

Okada, M., Nishio, W., Sakamoto, T., Uchino, K., Yuki, T., Nakagawa, A., et al. (2004). Prognostic Significance of Perioperative Serum Carcinoembryonic Antigen in Non-small Cell Lung Cancer: Analysis of 1,000 Consecutive 
Resections for Clinical Stage I Disease. Ann. Thorac. Surg. 78 (1), 216-221. doi:10.1016/j.athoracsur.2004.02.009

Ozeki, N., Fukui, T., Taniguchi, T., Usami, N., Kawaguchi, K., Ito, S., et al. (2014). Significance of the Serum Carcinoembryonic Antigen Level during the Follow-Up of Patients with Completely Resected Nonsmall-cell Lung Cancer. Eur. J. Cardio-Thoracic Surg. 45 (4), 687-692. doi:10.1093/ejcts/ezt424

Plebani, M., Basso, D., Navaglia, F., De Paoli, M., Tommasini, A., and Cipriani, A. (1995). Clinical Evaluation of Seven Tumour Markers in Lung Cancer Diagnosis: Can Any Combination Improve the Results? Br. J. Cancer 72, 170-173. doi:10.1038/bjc. 1995.296

Sawabata, N., Ohta, M., Takeda, S.-i., Hirano, H., Okumura, Y., Asada, H., et al. (2002). Serum Carcinoembryonic Antigen Level in Surgically Resected Clinical Stage I Patients with Non-small Cell Lung Cancer. Ann. Thorac. Surg. 74, 174-179. doi:10.1016/s0003-4975(02)03662-7

Sawabata, N., Maeda, H., Yokota, S., Takeda, S.-i., Koma, M., Tokunaga, T., et al. (2004a). Postoperative Serum Carcinoembryonic Antigen Levels in Patients with Pathologic Stage IA Nonsmall Cell Lung Carcinoma. Cancer 101, 803-809. doi:10.1002/cncr.20421

Sawabata, N., Maeda, H., Yokota, S., Takeda, S.-I., Koma, M., Tokunaga, T., et al. (2004b). Postoperative Serum Carcinoembryonic Antigen Levels in Patients with Pathologic Stage IA Nonsmall Cell Lung Carcinoma. Cancer 101 (4), 803-809. doi:10.1002/cncr.20421

Schneider, J., Velcovsky, H. G., Morr, H., Katz, N., Neu, K., and Eigenbrodt, E. (2000). Comparison of the Tumor Markers Tumor M2-PK, CEA, CYFRA 21-1, NSE and SCC in the Diagnosis of Lung Cancer. Anticancer Res. 20, 5053-5058.

Sung, H., Ferlay, J., Siegel, R. L., Laversanne, M., Soerjomataram, I., Jemal, A., et al. (2021). Global Cancer Statistics 2020: GLOBOCAN Estimates of Incidence and Mortality Worldwide for 36 Cancers in 185 Countries. CA Cancer J. Clin. 71, 209-249. doi:10.3322/caac. 21660

Suzuki, K., Nagai, K., Yoshida, J., Moriyama, E., Nishimura, M., Takahashi, K., et al. (1999). Prognostic Factors in Clinical Stage I Non-small Cell Lung Cancer. Ann. Thorac. Surg. 67 (4), 927-932. doi:10.1016/s00034975(99)00140-x

Takahashi, N., Suzuki, K., Takamochi, K., and Oh, S. (2011). Prognosis of Surgically Resected Lung Cancer with Extremely High Preoperative Serum Carcinoembryonic Antigen Level. Gen. Thorac. Cardiovasc. Surg. 59 (10), 699-704. doi:10.1007/s11748-011-0797-x

Takamochi, K., Yoshida, J., Nishimura, M., Yokose, T., Sasaki, S., Nishiwaki, Y., et al. (2004). Prognosis and Histologic Features of Small Pulmonary Adenocarcinoma Based on Serum Carcinoembryonic Antigen Level and
Computed Tomographic Findings. Eur. J. Cardio-Thoracic Surg. 25 (5), 877-883. doi:10.1016/j.ejcts.2004.01.049

Thomas, S. N., Zhu, F., Schnaar, R. L., Alves, C. S., and Konstantopoulos, K. (2008). Carcinoembryonic Antigen and CD44 Variant Isoforms Cooperate to Mediate Colon Carcinoma Cell Adhesion to E- and L-Selectin in Shear Flow. J. Biol. Chem. 283 (23), 15647-15655. doi:10.1074/jbc.M800543200

Tomita, M., Shimizu, T., Ayabe, T., Yonei, A., and Onitsuka, T. (2010). Prognostic Significance of Tumour Marker index Based on Preoperative CEA and CYFRA 21-1 in Non-small Cell Lung Cancer. Anticancer Res. 30 (7), 3099-3102.

Tomita, M., Ayabe, T., Chosa, E., and Nakamura, K. (2015). Postoperative Serum CEA Level Is a More Significant Prognostic Factor Than Post/Preoperative Serum CEA Ratio in Non-small Cell Cancer Patients. Asian Pac. J. Cancer Prev. 16 (17), 7809-7812. doi:10.7314/APJCP.2015.16.17.7809

Wang, C.-Y., Huang, M.-S., Huang, M.-H., Lee, H.-C., and Hsu, H.-S. (2010). Persistently High Serum Carcinoembryonic Antigen Levels After Surgery Indicate Poor Prognosis in Patients with Stage I Non-small-cell Lung Cancer. J. Surg. Res. 163 (2), e45-e50. doi:10.1016/j.jss.2010.04.039

Wang, X.-B., Li, J., and Han, Y. (2014). Prognostic Significance of Preoperative Serum Carcinoembryonic Antigen in Non-small Cell Lung Cancer: A MetaAnalysis. Tumor Biol. 35 (10), 10105-10110. doi:10.1007/s13277-014-2301-6

Yamazaki, M., Ishikawa, H., Kunii, R., Tasaki, A., Sato, S., Ikeda, Y., et al. (2015). A Combination of Preoperative CT Findings and Postoperative Serum CEA Levels Improves Recurrence Prediction for Stage I Lung Adenocarcinoma. Eur. J. Radiol. 84 (1), 178-184. doi:10.1016/j.ejrad.2014.10.009

Conflict of Interest: The authors declare that the research was conducted in the absence of any commercial or financial relationships that could be construed as a potential conflict of interest.

Publisher's Note: All claims expressed in this article are solely those of the authors and do not necessarily represent those of their affiliated organizations, or those of the publisher, the editors and the reviewers. Any product that may be evaluated in this article, or claim that may be made by its manufacturer, is not guaranteed or endorsed by the publisher.

Copyright $\odot 2021$ Jiao, Cao, Li, Hu, Gong, Wang and Jin. This is an open-access article distributed under the terms of the Creative Commons Attribution License (CC $B Y)$. The use, distribution or reproduction in other forums is permitted, provided the original author(s) and the copyright owner(s) are credited and that the original publication in this journal is cited, in accordance with accepted academic practice. No use, distribution or reproduction is permitted which does not comply with these terms. 